Descargo de responsabilidad: las opiniones expresadas en los manuscritos son responsabilidad exclusiva de los autores. No necesariamente reflejan las opiniones de la editorial ni la de sus miembros.

1 CENIKO (1)

\title{
Factores que dificultan el aprendizaje en pandemia de los niños preescolares
}

\section{Factors hindering pandemic learning in preschool children}

Carla Suyana Godínez Coz

suyana.godinez@yahoo.es

https://orcid.org/0000-0003-2426-138X

Recibido: $27 / 10 / 2021$

Publicado: 20/01/2022

\section{Filiación institucional de los autores}

Maestría en Neurociencia con énfasis en Neurocognición

Universidad de San Carlos de Guatemala
Eslia Yesenia Pérez Pernillo esliaperez@gmail.com https://orcid.org/0000-0001-7379-9421

Clara Marcela García García marcela16garcia@postgradocunzac.edu.gt https://orcid.org/0000-0001-6787-8799

Ingrid Irlanda Balán González

ingridbalanpadeptic@gmail.com

https://orcid.org/0000-0002-2051-3642

\section{Referencia}

Godínez Coz, C. S., Pérez Pernillo, E. Y., García García , C. M., \& Balán González, I. I. (2022). Factores que dificultan el aprendizaje en pandemia de los niños preescolares. Revista Académica Sociedad Del Conocimiento Cunzac, 2(1), 41-47. DOI: https://doi.org/10.46780/sociedadcunzac.v2i1.11

\section{Resumen}

OBJETIVO: identificar los factores sociofamiliares, emocionales, económicos, y tecnológicos que influyen en el proceso de aprendizaje en niños de edad preescolar. METODO: se utilizó la metodología mixta y recolectaron los datos por medio de entrevistas telefónicas dirigidas a las diez maestras del nivel preescolar de una escuela del departamento de Petén. RESULTADOS: entre los factores que dificultaron el aprendizaje el tecnológico fue el de mayor significancia con un $40 \%$ y de este el $70 \%$ no cuenta con dispositivo electrónico de comunicación. CONCLUSIÓN: la falta de dispositivos electrónicos para que el niño pudiera llevar a cabo su aprendizaje escolar se ve afectado ya que en algunos casos los padres solo cuentan con un dispositivo electrónico o no cuentan con los recursos para agenciarse de otro y dejarlo en casa mientras ellos salen a trabajar.

\section{Palabras clave}

pandemia, factores, aprendizaje, preescolar, neurodidáctica 


\section{Abstract}

OBJECTIVE: To identify the socio-familial, emotional, economic, and technological factors that influence the learning process in preschool children. METHOD: A mixed methodology was used and data were collected by means of telephone interviews with ten preschool teachers of the Official Nursery School "Rosa María Carlota Tello Aguilar de Castillo" in the department of Petén. RESULTS: Among the factors that hindered learning, the technological factor was the most significant with $40 \%(\mathrm{~N}=10)$ and $70 \%$ of these did not have an electronic communication device. CONCLUSION: The lack of electronic devices so that children could carry out their school learning is affected because in some cases parents only have one electronic device or do not have the resources to get another one and leave it at home while they go out to work.

\section{Keywords}

pandemic, factors, learning, preschool, neurodidactics

\section{Introducción}

Hablar de la educación preescolar, es hablar de niños de 4 a 6 años. Según el último Censo (INE 2018) poblacional en Guatemala a nivel nacional son 625,829 niños que se encuentran en dicha etapa educativa, el departamento de Petén tiene una población de 25,465 niños de 4 a 6 años. La muestra de estudio fueron las diez secciones del nivel preescolar con las que cuenta la escuela. Este nivel educativo es fundamental en el desarrollo del niño, en esa etapa su sistema nervioso está en formación, su psiquismo en construcción y su personalidad en elaboración.

A finales del año 2019 se tuvo conocimiento de la aparición del SARSCov2 o Coronavirus que genera la enfermedad Covid-19, se dio el fenómeno natural de creer que estaba tan lejos que tal vez no nos afectaría, pero producto de la globalización, la expansión del comercio y la facilidad de transportarse que existe en la actualidad, llegó a al país en marzo de 2020 . Fue entonces cuando se empezó a vivir con más intensidad la pandemia, impactando a todo nivel, incluida la educación.

El papel del maestro es esencial en la formación de la niñez, deberá tener una participación inclusiva y activa. El aprendizaje a través del juego involucra las emociones, la motivación y la atención, que es fundamental en el proceso de enseñanza aprendizaje y son los fundamentos de la neurodidáctica. No obstante, aunque el maestro propicie un ambiente agradable que favorezca el aprendizaje, prepare actividades innovadoras y aplique sus conocimientos de neurodidáctica, sus esfuerzos pueden no ser suficientes, debido a factores externos que dificultan el aprendizaje; se identificaron los siguientes: sociofamiliares, emocionales, económicos y tecnológicos. Estos se agudizaron por la pandemia derivada del Covid-19.

El papel de los padres es fundamental para que el proceso de enseñanza aprendizaje se dé de la mejor manera. Algunos padres se enfocan en que el niño saque buenas notas, aprenda a leer, escribir, hacer operaciones matemáticas básicas; restándole valor a otros aspectos importantes del proceso formativo de la etapa preescolar, que se desarrolla a través del juego espontáneo, la convivencia, el aprendizaje cooperativo, actividades lúdicas dirigidas y artísticas. 


\section{Materiales y métodos}

Se realizó un estudio de diseño descriptivo transversal, midiendo las características socio demográficas de la muestra, en la escuela durante la pandemia por COVID 19, con 249 niños de edad preescolar por medio de recolección de datos previamente realizando un consentimiento informado por los padres de familia, docentes y dirección del plantel educativo. Se recolecto la información de características sociodemográficas, factores que intervienen en el aprendizaje y las causas de los factores de dificultad de aprendizaje consecuencias que fueron las desencadenantes. A partir de ello se plantea realizar dos talleres formativos, uno para maestros y uno para padres de familia.

\section{Resultados}

Tabla 1. Característica Sociodemográfica.

\begin{tabular}{llcc}
\hline Sub- variables & $\begin{array}{c}\text { Cantidad (expresado en } \\
\text { números) }\end{array}$ & $\begin{array}{c}\text { Cantidad (expresado en } \\
\% \text { ) }\end{array}$ \\
\cline { 2 - 4 } Edad & de 4 a 6 años & $\mathbf{2 4 9}$ & $\mathbf{1 0 0 \%}$ \\
\hline \multirow{2}{*}{ Sexo } & Niñas & 126 & $51 \%$ \\
\cline { 2 - 4 } & Niños & 123 & $49 \%$ \\
\hline \multirow{2}{*}{ Tipo de familia } & & $\mathbf{2 4 9}$ & $\mathbf{1 0 0 \%}$ \\
\cline { 2 - 4 } & Integrada & 224 & $90 \%$ \\
\cline { 2 - 4 } & Desintegrada & 25 & $10 \%$ \\
\hline \multirow{2}{*}{ Etnia } & & $\mathbf{2 4 9}$ & $\mathbf{1 0 0 \%}$ \\
\cline { 2 - 4 } & Ladino & 236 & $95 \%$ \\
\cline { 2 - 4 } & Maya & 11 & $4 \%$ \\
\cline { 2 - 4 } & Mestizo & 2 & $0 \%$ \\
\cline { 2 - 4 } & Otros & 0 & $1 \%$ \\
\hline
\end{tabular}

Fuente: elaboración propia con base a la entrevista telefónica estructurada.

En la tabla 1, se observan las características sociodemográficas de los niños de la escuela que se encuentran actualmente estudiando. De los 249 niños de edad preescolar, 126 son niñas con el 51\%, mientras que 123 son niños representando el 49\%. En su mayoría 224 niños provienen de familia integrada en $90 \%$ y solo 25 niños de familia desintegrada en un $10 \%$. En cuanto a la etnia 236 son ladinos constituyendo el $95 \%$, de la etnia maya son 11 con un $4 \%$ y solamente 2 mestizos que representan el $1 \%$ es mestizo. 
Tabla 2. Factores que dificultan el aprendizaje.

\begin{tabular}{lcc}
\hline \multirow{2}{*}{ Factores } & $\begin{array}{c}\text { Cantidad (expresadoen } \\
\text { números) }\end{array}$ & $\begin{array}{c}\text { Cantidad (expresadoen } \\
\%)\end{array}$ \\
\cline { 2 - 3 } & 10 & $100 \%$ \\
\hline Tecnológicos & 4 & $40 \%$ \\
\hline Sociofamiliares & 3 & $30 \%$ \\
\hline Económicos & 2 & $20 \%$ \\
\hline Emocionales & 1 & $10 \%$ \\
\hline
\end{tabular}

Fuente: elaboración propia con base a la entrevista telefónica estructurada.

Los resultados de la tabla 2, evidencian que de 10 maestras entrevistadas, 4 coincidieron con el factor tecnológico (40\%), seguido por el factor sociofamiliar con un 30\% (3 maestras), en los factores económicos 2 de las maestras con $20 \%$ y por último

Tabla No. 3. Causas de los factores de dificultad de aprendizaje.

\begin{tabular}{|c|c|c|c|c|c|}
\hline Factores & Cantidad & Porcentaje & & Cantidad & Porcentaje \\
\hline \multirow[t]{5}{*}{ Sociofamiliares } & 10 & $100 \%$ & $\begin{array}{l}\text { Poco apoyo por parte de } \\
\text { los padres }\end{array}$ & 7 & $78 \%$ \\
\hline & & & Desintegración familiar & 2 & $22 \%$ \\
\hline & & & Violencia intrafamiliar & 0 & $0 \%$ \\
\hline & & & Adicciones & 0 & $0 \%$ \\
\hline & & & Hacinamiento & 0 & $0 \%$ \\
\hline \multirow[t]{3}{*}{ Económicos } & 10 & $100 \%$ & Desempleo & 9 & $90 \%$ \\
\hline & & & Pobreza & 1 & $10 \%$ \\
\hline & & & No cuenta con dispositivo & & \\
\hline \multirow[t]{4}{*}{ Tecnológicos } & 10 & $100 \%$ & $\begin{array}{l}\text { electrónico de } \\
\text { comunicación }\end{array}$ & 7 & $70 \%$ \\
\hline & & & Falta de acceso a internet & 6 & $60 \%$ \\
\hline & & & Mala conectividad & 4 & $40 \%$ \\
\hline & & & $\begin{array}{l}\text { Desconoce el uso de las } \\
\text { herramientas tecnológicas }\end{array}$ & 4 & $40 \%$ \\
\hline \multirow[t]{7}{*}{ Emocionales } & 9 & $90 \%$ & Ansiedad & 5 & $56 \%$ \\
\hline & & & Aislamiento & 3 & $33 \%$ \\
\hline & & & Desinterés & 2 & $22 \%$ \\
\hline & & & Timidez & 2 & $22 \%$ \\
\hline & & & Tristeza & 2 & $22 \%$ \\
\hline & & & Miedo & 2 & $22 \%$ \\
\hline & & & Agresividad & 2 & $22 \%$ \\
\hline
\end{tabular}

Fuente: elaboración propia con base a la entrevista telefónica estructurada. 
En la tabla 3 se muestran las respuestas que cada una de las maestras respondió por cada una de las causas de los factores de dificultad de aprendizaje, el poco apoyo por parte de los padres obtuvo el $78 \%$ (7 maestras) del factor sociofamiliar, seguido con un $22 \%$ ( 2 maestras) desintegración familiar.

En el económico se evidencio el desempleo con un 90\% (9 maestras) y con $1 \%$ la pobreza (1 maestra), las causas tecnológicas específicamente el no contar con un dispositivo electrónico de comunicación se encuentra con un 70\% (7 maestras respondieron que es una de las limitantes para el proceso de aprendizaje), estas tres causas se asocia que la pandemia trajo consigo el desempleo y los padres tuvieron que buscar la forma de generar ingresos, descuidando las tareas escolares de sus hijos y no apoyándolos de la manera que lo hacían anteriormente.

Se pudo observar en la tabla que otra de las causas que dificulta el aprendizaje es la falta de acceso a internet con un $60 \%$ que representa 6 maestras, esto ha ocasionado que algunos estudiantes no puedan acceder a las clases y no cumplan con las tareas asignadas. La mala conectividad y el desconocimiento de las herramientas tecnológicas son otras de las circunstancias que obstaculiza dicho proceso con un $40 \%$ cada una (4 maestras). Aunado a esto algunas familias solo cuentan con un dispositivo electrónico de comunicación que las mayorías de las veces es de los cuidadores que llevan el sustento diario.

Según Rotger (2018), indica que "las emociones son reacciones psicofisiológicas que representan modos de adaptación a ciertos estímulos del individuo cuando percibe un objeto, persona, lugar, suceso o recuerdo importante", es por ello por lo que se tomó en cuenta las causas emocionales. Cabe mencionar que, de las 10 maestras, solo respondieron 9, ya que 1 se abstuvo de responder. Así mismo, las maestras describieron distintas causas a raíz del confinamiento, que al dar sus clases pudieron percibir ansiedad en los niños, siendo el $56 \%$ de ellos (5 maestras). El aislamiento con el 33\% (3 maestras), el desinterés, timidez, tristeza y miedo con $22 \%$ cada uno ( 2 maestras).

Estas causas pueden intervenir en la enseñanza-aprendizaje a todo nivel, todos respondemos ante las demandas de acuerdo con nuestros aprendizajes, para muchas personas fue agradable y/o manejable el confinamiento, pero para otras personas significó tener que convivir con quienes generan malestar emocional y pasado el tiempo sentirse sobrepasados por la convivencia.

\section{Discusión}

Según datos de la Organización de las Naciones Unidas para la Educación, la Ciencia y la Cultura (UNESCO) "a mediados de mayo 2020 más de 1,200 millones de estudiantes de todos los niveles de enseñanza, en todo el mundo, habían dejado de tener clases presenciales en la escuela. De ellos más de 160 millones eran estudiantes de América Latina y el Caribe" (UNESCO, 2020).

Los hallazgos de la presente investigación evidencian que los factores que dificultan el aprendizaje, en orden de prevalencia son: tecnológicos con el $40 \%$, debido a la falta de dispositivos 
electrónicos 70\%; los sociofamiliares con el $30 \%$, por el poco apoyo de los padres $78 \%$; los económicos con el $20 \%$, por el desempleo $90 \%$ y los emocionales con el $10 \%$, por la ansiedad $56 \%$.

La falta de dispositivos electrónicos para que el niño pudiera llevar a cabo su aprendizaje escolar se ve afectado ya que en algunos casos los padres solo cuentan con un dispositivo electrónico o no cuentan con los recursos para agenciarse de otro y dejarlo en casa mientras ellos salen a trabajar. Dificultando así el aprendizaje.

El poco apoyo de los padres está asociado a que el trabajo fue llevado a casa y la jornada laboral aumento. En algunos casos en los que hubo desempleo, priorizaron la búsqueda de fuentes de ingreso para sus hogares.

Aunque se piense que la etapa preescolar no es importante o necesaria para el desarrollo integral del niño, los estudios de neurociencia están contribuyendo de manera significativa en el conocimiento y desarrollo del cerebro. La neurodidáctica que es una rama de la pedagogía, basada en las neurociencias, que involucra la psicología, ambas enfocadas en el arte y la ciencia de enseñar, incrementando los procesos mentales responsables de la cognición y el comportamiento. Su objetivo es otorgar respuestas a la diversidad de alumnos, atendiendo a las distintas modalidades de aprendizaje, sus fundamentos son la emoción, la atención y la motivación.

Esta disciplina ofrece grandes oportunidades de cambio en el cerebro debido a la cantidad de estímulos que el estudiante recibe en el entorno educativo, sin embargo, también tiene limitaciones entre ellas, los factores tecnológicos y económicos.

\section{Referencias}

CEPAL - UNESCO. (2020). La educación en tiempos de la pandemia de COVID-19. Naciones Unidas. DOI: https://doi.org/10.24875/.j.gamo.M20000200

Instituto Nacional de Estadística de Guatemala INE. (2018). "Portal de resultados del ya Censo 2018." www.censopoblacion.gt.

Rotger M. (2018). Neurociencias, Neuroaprendizaje: Las emociones y el aprendizaje. https:// es.scribd.com/document/525977773/Neurociencias-Neuroaprendizaje-Marilina-Rotger

\section{Sobre las autoras}

Grupo de investigadoras de la Maestría de Neurociencias con énfasis en neurocognición en el Centro Universitario de Zacapa de la Universidad de San Carlos de Guatemala. 


\section{Financiamiento de la investigación}

Con recursos propios de los investigadores

\section{Declaración de intereses}

Declaran no tener ningún conflicto de intereses, que puedan haber influido en los resultados obtenidos o las interpretaciones propuestas.

\section{Declaración de consentimiento informado}

El estudio se realizó respetando el Código de ética y buenas prácticas editoriales de publicación.

\section{Derechos de uso}

Copyright@ 2022 por Carla Suyana Godínez Coz, Eslia Yesenia Pérez Pernillo, Clara Marcela García García y Ingrid Irlanda Balán González

Este texto está protegido por una licencia Creative Commons 4.0

Este texto está protegido por la Licencia Creative Commons Atribución 4.0 Internacional.

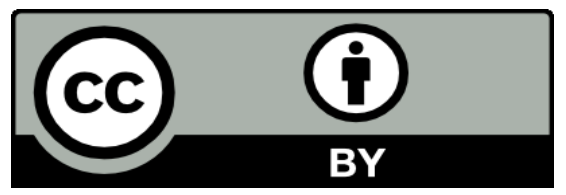

Este texto está protegido por una licencia

Creative Commons 4.0.

Es libre para compartir, copiar y redistribuir el material en cualquier medio o formato y adaptar el documento, remezclar, transformar y crear a partir del material para cualquier propósito, incluso comercialmente, siempre que cumpla la condición de atribución: debe reconocer el crédito de una obra de manera adecuada, proporcionar un enlace a la licencia, e indicar si se han realizado cambios. Puede hacerlo en cualquier forma razonable, pero no de forma tal que sugiera que tiene el apoyo del licenciante o lo recibe por el uso que hace. 